\title{
Refraction of Water Waves by Periodic Cylinder Arrays
}

\author{
Xinhua $\mathrm{Hu}^{*}$ and C. T. Chan \\ Department of Physics, Hong Kong University of Science and Technology, Clear Water Bay, Kowloon, Hong Kong, China
}

(Received 14 December 2004; published 4 October 2005)

\begin{abstract}
We show that in the long wavelength limit, water waves propagate through an array of bottom-mounted vertical cylinders as if the water has an effective depth and effective gravitational constant that depends on the filling ratio of the cylinders, leading to refraction phenomena that can be described by analytic formulas. The results are obtained with rigorous homogenization techniques, as well as the multiple scattering formalism that gives full dispersion relationships. This phenomenon provides a mechanism to control the flow of ocean wave energy, as exemplified by a water-wave focusing lens.
\end{abstract}

DOI: 10.1103/PhysRevLett.95.154501

PACS numbers: 47.35.+i, 04.30.Nk, 92.20.Gr

The interaction of water waves with periodic structures, such as rippled bottoms, periodic cylinder arrays, and periodic arrays of surface scatters, has received considerable attention [1-13]. Most of the interest is stimulated by the discovery of the Bragg resonance of water waves when the periodic length is a multiple of the half-wavelength, leading to a huge reflection of water waves by a finite periodic structure [1,2]. This phenomenon is not only important to the understanding of the formation of periodic sand bars near beaches, but also provides a mechanism for coastal protection. Consequently, more general theories and powerful methods have been proposed and implemented for the water-wave studies over uneven bottom topographies $[3,4]$ and in vertical obstacle structures $[5,6]$.

On the other hand, inspired by the rapid progress in the field of photonic crystals [14], the technique of the Bloch theorem has been applied recently to solve the dispersion relations (band structures) of water waves in periodic structures [7-11]. It is found that there exist some frequency ranges in which wave propagations are forbidden, giving rise to water-wave band gaps. The dispersions near the band gaps are also greatly modified by the periodicity. Based on the band structures of water waves, more intriguing phenomena such as the instabilities of periodically sheared fluid [7], Bloch waves and domain walls over drilled bottoms [8], and the superlensing effect [12] were found.

While the frequency regime near the Bragg resonance has attracted most attention, there have been few studies on the long wave ranges far below the Bragg resonance [13]. In this Letter, we study the influence of an array of vertical bottom-mounted cylinders on the propagation of long water waves. For long waves (wavelength $\gg$ lattice constant $a$ ), we find that the periodic cylinder array system behaves like an effective medium which has an effective depth $h_{e}$ and a renormalized gravitational constant $g_{e}[15,16]$. We are able to derive analytic formulas for $h_{e}$ and $g_{e}$, and these formulas are very useful in predicting refraction, reflection, or transmission results and we also found the existence of a Brewster angle. The accuracy of these formulas is confirmed by dispersion relation calculations as well as numerical simulations using multiple scattering techniques.

Our system has also its acoustic/electromagnetic (EM) analogue [17], where an empirical formula was found for the effective sound/light velocity $v_{e}[18,19]$. However, no analytical derivation is given. In addition, most of these approaches focus on finding $v_{e}$. For example, $\kappa_{e}=\kappa_{\text {air }}$ has been presumed in a simple model for the acoustic wave analogue $[17,19]$. We stress the importance that in a $2 \mathrm{D}$ problem, two constitutive parameters should be homogenized in the same time and that comes naturally from our formulation.

We consider linear, inviscid, and irrotational water waves in infinite extent of water of constant depth $h$, pierced with a square lattice of identical, vertical, rigid, circular cylinders as shown in Fig. 1(a). Set $\mathbf{r}=(x, y)$ in the horizontal plane and $z$ as the vertical axis. For harmonic water waves with angular frequency $\omega$, the vertical displacement of the water surface $\eta(\mathbf{r}) e^{i \omega t}$ obeys the two-

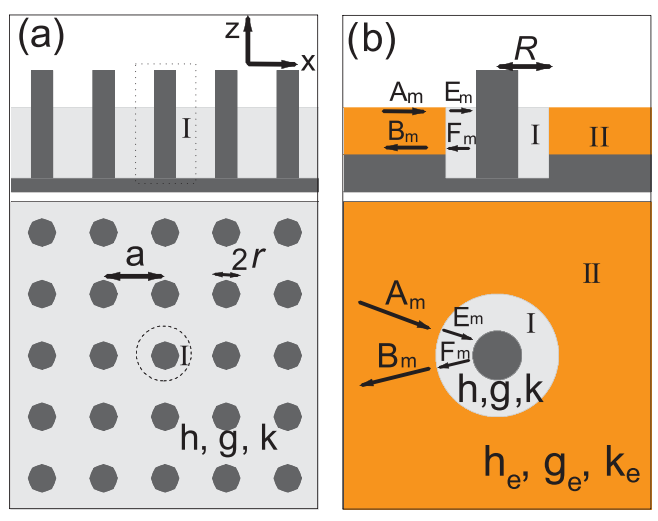

FIG. 1 (color online). Schematic diagrams of a periodic array of identical, vertical, rigid cylinders standing in water with constant depth $h$ (a), and a water column pierced by a vertical rigid cylinder, which is surrounded by an "effective" water medium (b). The top panels are the side views and the bottom panels are the vertical views. 
dimensional Helmholtz equation $[5,6,10-12,20]$ :

$$
\left(\nabla^{2}+k^{2}\right) \eta=0
$$

which is subjected to the boundary condition of no flow through the cylinder walls, namely

$$
\frac{\partial \eta}{\partial \mathbf{n}}=0
$$

at the surface of each cylinder, where $\mathbf{n}$ is the direction normal to the cylinder surface. The wave number $k$ can be obtained from the dispersion relation $\omega^{2}=g k \tanh (k h)$, where $g$ is the gravitational acceleration.

We will first derive analytical formulas for $h_{e}$ and $g_{e}$ with the coherent-potential-approximation (CPA) method $[16,21,22]$ in the long wave limit with $k a \ll 1$. We consider a circular water column [23] with radius $R=a / \sqrt{\pi}$ (so that $\frac{\pi r^{2}}{\pi R^{2}}$ equals the filling fraction $f_{s}=\frac{\pi r^{2}}{a^{2}}$ ), water depth $h$, and pierced by a rigid circular cylinder with radius $r$, surrounded by the effective medium as shown in Fig. 1(b). To deal with the case with uneven bottom, we use the shallow water-wave equation (rigorous when $k h \ll 1$ ) $[3,4,9,20]$ :

$$
\nabla(h \nabla \eta)+\frac{\omega^{2}}{g} \eta=0,
$$

which implies a linear dispersion of $\omega=\sqrt{g h} k$ and continuities of $h \frac{\partial \eta}{\partial \rho}$ and $\eta$. Using the cylindrical coordination $(\rho, \phi)$ with the origin at the center of cylinder, $\eta$ can be written as

$$
\begin{aligned}
\eta^{\mathrm{I}} & =\sum_{m}\left[E_{m} J_{m}(k \rho)+F_{m} H_{m}(k \rho)\right] e^{i m \phi} \quad \text { when } R \geq \rho \geq r, \\
\eta^{\mathrm{II}} & =\sum_{m}\left[A_{m} J_{m}\left(k_{e} \rho\right)+B_{m} H_{m}\left(k_{e} \rho\right)\right] e^{i m \phi} \quad \text { when } \rho \geqslant R,
\end{aligned}
$$

where the Bessel-Hankel function $J_{m} / H_{m}$ stands for the incident/scattering waves, $k_{e}$ is the wave number for the effective medium, and $k$ for the water column. Using $\frac{\partial \eta^{\mathrm{I}}(r)}{\partial \rho}=0, \quad \eta^{\mathrm{I}}(R)=\eta^{\mathrm{II}}(R)$, and $h \frac{\partial \eta^{\mathrm{I}}(R)}{\partial \rho}=h_{e} \frac{\partial \eta^{\mathrm{II}}(R)}{\partial \rho}$, the scattering coefficient $D_{m}=\frac{B_{m}}{A_{m}}$ of the water column for the $m$ th-order cylindrical incident waves can be obtained and $D_{m}=0$ (which "defines" the effective medium) when

$$
\begin{aligned}
0= & -h k J_{m}\left(k_{e} R\right)\left[H_{m}^{\prime}(k R) J_{m}^{\prime}(k r)-J_{m}^{\prime}(k R) H_{m}^{\prime}(k r)\right] \\
& +h_{e} k_{e} J_{m}^{\prime}\left(k_{e} R\right)\left[H_{m}(k R) J_{m}^{\prime}(k r)-J_{m}(k R) H_{m}^{\prime}(k r)\right] .
\end{aligned}
$$

When $k a \ll 1$, Eq. (4) becomes as

$$
\begin{gathered}
h_{e} k_{e}^{2}=\left(1-f_{s}\right) h k^{2} \quad \text { for } m=0, \\
h_{e}=h \frac{1-f_{s}^{m}}{1+f_{s}^{m}} \quad \text { for } m \geq 1 .
\end{gathered}
$$

Using $k_{e}=\frac{\omega}{\sqrt{g_{e} h_{e}}}$ and $k=\frac{\omega}{\sqrt{g h}}$, the nonreflection condition for the zeroth-order and first-order cylindrical incident waves can be written, respectively, as

$$
g_{e}=\frac{1}{1-f_{s}} g, \quad h_{e}=\frac{1-f_{s}}{1+f_{s}} h .
$$

For 1D model system with periodic uneven bottom, $g_{e}=g$ for water waves $[13,16]$, but in 2D, the $g_{e}$ is renormalized. Besides, the dispersion in the effective medium can be obtained as $k_{e}=n_{e} k$, where

$$
n_{e}=\sqrt{1+f_{s}} .
$$

We note that Eq. (7) has appeared in acoustics [19], but has been interpreted with a model with $\kappa_{e}=\kappa_{\text {air }}$ and $\rho_{e}=$ $\left(1+f_{s}\right) \rho_{\text {air }}$. The correct homogenization should involve both parameters as in Eq. (6).

This effective $n_{e}$ can be used to determine refraction angle and Brewster angle. Then we consider the incidence of a long plane water wave from the water with $g$ and $h$ onto the effective water with $g_{e}$ and $h_{e}$ as shown in Fig. 2. Conservation of the parallel wave vector component at the interface leads to

$$
\sin \theta=n_{e} \sin \varphi
$$

where $\theta$ and $\varphi$ are the incident and refractive angles, respectively. Using the continuity of $\eta$ and $h \frac{\partial \eta}{\partial y}$, the amplitude reflection coefficient $r_{A}$ and the amplitude transmission coefficient $t_{A}$ can be obtained as

$$
r_{A}=\frac{\cos \theta-Z \cos \varphi}{\cos \theta+Z \cos \varphi}, \quad t_{A}=\frac{2 \cos \theta}{\cos \theta+Z \cos \varphi},
$$

where $Z=\sqrt{g h_{e} /\left(g_{e} h\right)}=\left(1-f_{s}\right) / \sqrt{1+f_{s}}$. So when $\theta=\frac{\pi}{2}, r_{A}=-1$, leading to a complete reflection. When

$$
\theta=\theta_{0}=\arccos \frac{1-f_{s}}{2},
$$

the reflection will be zero (Brewster angle). When $\theta=\theta_{0}$, $2 \theta+\varphi=\pi$.

The solution of Eqs. (1) and (2) is independent of $h$ (only dependent on $k$ and the geometry of cylinders). So, Eqs. (7)-(10) will be valid in any $h$ (only limited by $k a \ll$ 1) although they are obtained under $k h \ll 1$. This means that the refraction of water waves by the periodic cylinder array can occur in any depth, in contrast with the common refraction of water waves by varying depth which can only occur in the shallow water.

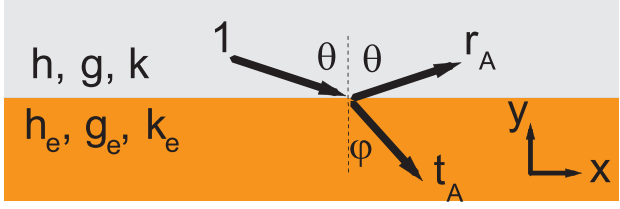

FIG. 2 (color online). Reflection and refraction of a plane water wave by an effective water medium. 
To check the validity of the analytic formulas above, we use the multiple scattering (MS) method [5,6,11,12], which gives numerical results for Eqs. (1) and (2) for any $k$. In periodic structures, $\eta(\mathbf{r})=e^{i \mathbf{Q} \cdot \mathbf{r}} u(\mathbf{r})$, where $\mathbf{Q}$ is the Bloch wave vector and $u(\mathbf{r})$ is a two-dimensional periodic function. Based on a new fast-convergence technique for lattice sums, the dispersion relation $k(\mathbf{Q})$ can be accurately calculated [11]. We find that the dispersions are isotropic and linear when $k a<0.5 \pi$ and thus a refractive index $n_{d}$ can be defined: $n_{d}=|\mathbf{Q}| / k[18,19]$. In Fig. 3, we compare the refractive indexes of $n_{d}$ and $n_{e}$ for the long water waves in periodic cylinder arrays with different filling fractions $f_{s}$. It can be seen that $n$ increases with increasing filling fraction and $n_{e}$ agrees well with $n_{d}$. When $f_{s}>0.5$, the scattering of high-order cylindrical waves becomes important and the effective medium formula will deviate from the accurate values.

To further verify our results, and to take us beyond the long wavelength limit, we do MS simulations for the incidence of a plane water wave with wavelength $\lambda=$ $5 a(k a=0.4 \pi)$ on a ten-layer cylinder array with radius $r=0.35 a$ extending to infinity in the $x$ direction. The wave patterns for different incident angles $\theta$ of $20^{\circ}, 40^{\circ}$, $60^{\circ}, 80^{\circ}$ are shown in Figs. 4(a)-4(d). Above the cylinder array, some nodes can be seen due to the interference of incident and reflection waves. Inside the cylinder array, the transmitted waves have a well-defined wave front, showing refraction with refraction angle $\varphi$ determined as $17^{\circ}, 32^{\circ}$, $46^{\circ}$, and $54^{\circ}$, respectively. We found that an isotropic refractive index of $n_{r}=1.19$, satisfying the Snell law $\sin \theta=n_{r} \sin \varphi$, can be obtained for all the incident angles. When the water waves encounter the bottom side of the cylinder array, they will refract again and become parallel to the initial incidence. In Fig. 3, we plot the refractive index $n_{r}$ (dots) for different $f_{s}$, agreeing well with the above analytic values and dispersion calculations.

In Fig. 4(e), we show the reflectance vs incident angle $\theta$ for a nine-layer cylinder array. We have found that the reflection can be zero when $\theta=32^{\circ}$ or $68^{\circ}$ and the water waves can pass through the cylinder arrays completely. The

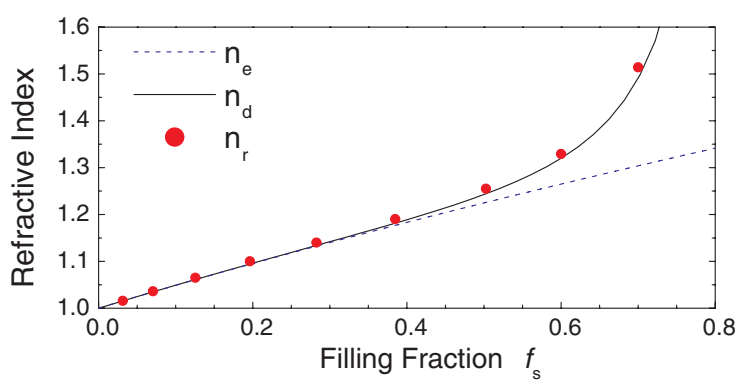

FIG. 3 (color online). The water-wave refractive index of periodic cylinder arrays as a function of the filling fraction. $n_{e}$ is determined from Eq. (7), $n_{d}$ is determined from dispersion calculations, and $n_{r}$ is determined by examining the wave fronts of plane wave in Figs. 4(a)-4(d). first reflection dip shifts with varying the thickness of the cylinder array and is due to the Fabry-Perot effect. The second reflection dip will persist at $\theta=68^{\circ}$ when the thickness of the cylinder array is varied, agreeing well with the Brewster angle of $\theta_{0}=72^{\circ}$ predicted by the effective medium theory of Eq. (10). Note that the Brewster angle phenomenon exists in optics for the $p$ polarization incidence. Zero reflection when $\theta=\frac{\pi}{4}$ (here $\theta_{0}=\frac{\pi}{3}$ when $f_{s} \rightarrow 0$ ) has also been found in refraction of water waves when the depth is slightly varied [2]. It is the first time that the Brewster angle phenomenon is found for water waves in cylinder arrays (also for acoustics/EM), and it also illustrates the usefulness of the effective medium formulas.

By now we have shown that the refraction of water waves by the cylinder arrays can occur in any water depth and will obey the Snell refractive law, with effective index given by Eq. (7) when $k a<0.5 \pi$. Many concepts in refractive optics can be applied to design the refractive devices for water waves based on such array structures. Water-wave focusing may make an impact on the electric generation with the ocean wave energy (EGOWE) [24]. In many situations, the efficiency and application of EGOWE is limited by the small wave amplitude. The cylinder structures can be used to focus water-wave energy [25], and thus increase the conversion efficiency.

In Fig. 5, we demonstrate the focusing of a plane water wave with $\lambda=5 a$ and with intensity of unit 1 by a biconvex periodic array of 657 cylinders with $r=0.35 a$. The plane water wave is incident from the left hand side of the cylinder array. The spatial pattern of transmitted intensity is shown in Fig. 5(a) and the focusing of the transmitted waves is evident. To quantitatively clarify the focusing

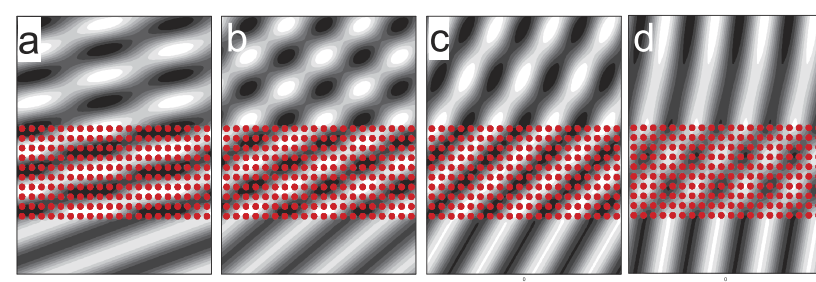

(e)

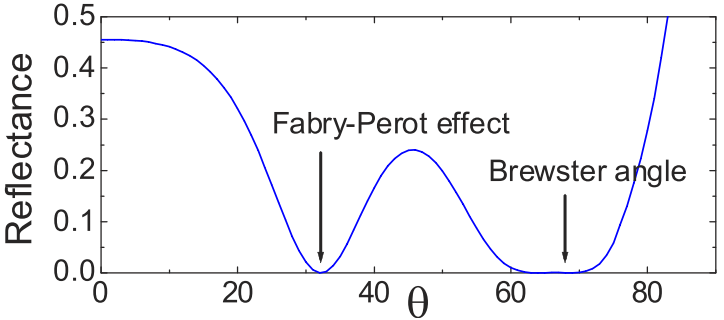

FIG. 4 (color online). Incidence of a plane water wave with wavelength $\lambda=5 a$ on cylinder arrays with $r=0.35 a$ : wave patterns for different incident angles of $20^{\circ}$ (a), $40^{\circ}$ (b), $60^{\circ}$ (c), $80^{\circ}$ (d) for a ten-layer cylinder array, and the reflectance as a function of the incident angle $\theta$ for a nine-layer cylinder array (e). 


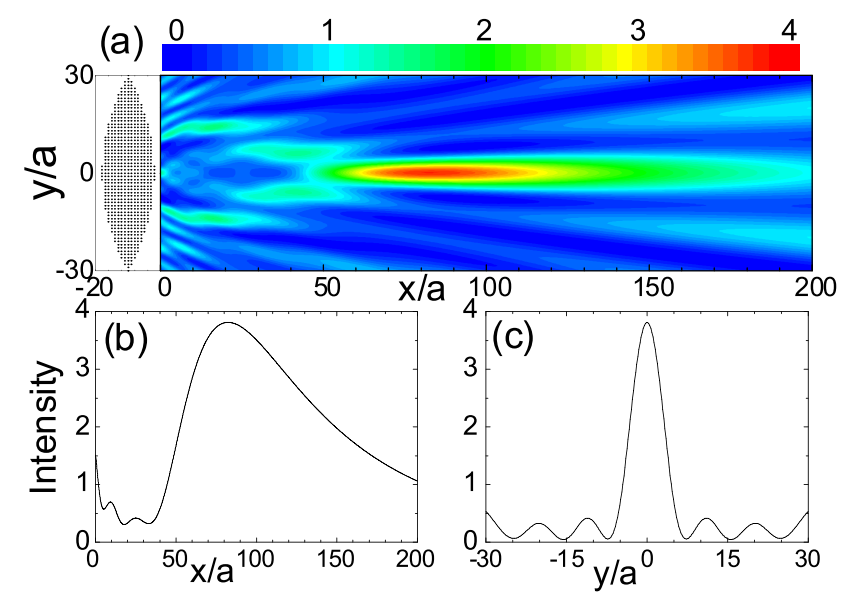

FIG. 5 (color online). Focusing of a plane water wave with $\lambda=5 a$ and with intensity of unit 1 by a biconvex periodic array of 657 cylinders with $r=0.35 a$ : (a) the spatial pattern of transmitted intensity, (b) the variation of the intensity along the $x$ axis at $y=0$, and (c) the variation of the intensity along the $y$ axis at $x=82.4 a$.

effect, the intensity along the $x$ axis is plotted in Fig. 5(b). We can see a maximum of intensity of 3.8 at $x=82.4 a$ and $y=0$. The intensity along the $y$ axis at $x=82.4 a$ is shown in Fig. 5(c), showing a transverse full width at half maximum of $6.8 a$ and $44 \%$ of wave energy within $|x|<30 a$ can be focused into a small area of $|x|<5 a$. The width of focus is already comparable to the wavelength and the focusing effect can, of course, be enhanced with a wider aperture device.

In summary, we have demonstrated that the long water waves propagate through an array of bottom-mounted cylinders as if it has an effective depth and gravitational constant [given by Eq. (6)], and we find an effective refractive index which can be used with the Snell refractive law and predicts a Brewster angle. This phenomenon can be used to control the flow of ocean wave energy and we present a conceptual design for a water-wave focusing lens based on this idea.

The authors thank Prof. Jian Zi, Dr. Jensen Li, and Dr. Yifeng Shen for interesting discussions. This work is supported by Hong Kong RGC through CA02/03.SC01.

*Present address: Department of Physics and Astronomy, Iowa State University, Ames, IA 50011, USA.

[1] A. D. Heathershaw, Nature (London) 296, 343 (1982); J. T. Kirby and R. A. Dalrymple, J. Fluid Mech. 133, 47 (1983); M. Naciri and C. C. Mei, J. Fluid Mech. 192, 51 (1988); M. Fernyhough and D. V. Evans, J. Fluid Mech. 305, 263 (1995).

[2] C. C. Mei, J. Fluid Mech. 152, 315 (1985).

[3] J. T. Kirby, J. Fluid Mech. 162, 171 (1986); B. Li, Coastal Engineering 23, 227 (1994); Y. Agnon, Phys.
Rev. E 59, R1319 (1999); Z. Ye, Phys. Rev. E 67, 036623 (2003).

[4] M. W. Dingemans, Water Wave Propagation over Uneven Bottoms (World Scientific, Singapore, 1997).

[5] C. Linton and D. Evans, J. Fluid Mech. 215, 549 (1990); R. Porter and D. Evans, Wave Motion 23, 95 (1996).

[6] Y. K. Ha et al., Appl. Phys. Lett. 81, 1341 (2002); T. S. Jeong et al., Appl. Phys. Lett. 85, 1645 (2004).

[7] T. Chou, Phys. Rev. Lett. 79, 4802 (1997); T. Chou, J. Fluid Mech. 369, 333 (1998).

[8] M. Torres et al., Nature (London) 398, 114 (1999); M. Torres et al., Phys. Rev. E 63, 011204 (2001).

[9] X. Hu et al., Phys. Rev. E 68, 066308 (2003); L.-S. Chen et al., Phys. Rev. E 69, 066308 (2004).

[10] P. McIver, J. Fluid Mech. 424, 101 (2000).

[11] X. Hu et al., Phys. Rev. E 68, 037301 (2003).

[12] X. Hu et al., Phys. Rev. E 69, 030201(R) (2004).

[13] R. Rosales and G. Papanicolaou, Stud. Appl. Math. 68, 89 (1983); A. Nachbin, J. Fluid Mech. 296, 353 (1995).

[14] E. Yablonovitch, Phys. Rev. Lett. 58, 2059 (1987); S. John, Phys. Rev. Lett. 58, 2486 (1987); For a recent review, see T.F. Krauss and R. M. De La Rue, Prog. Quantum Electron. 23, 51 (1999).

[15] N.S. Bakhvalov and G.P. Panasenko, Homogenization Averaging Process in Periodic Media (Kluwer, New York, 1989).

[16] The effective parameters can be obtained by the classical homogenization theory [15]. For a 1D system with a periodic uneven bottom that obeys Eq. (3), $g_{e}=g$ and $\frac{1}{h_{e}}=\left\langle\frac{1}{h}\right\rangle$. But numerical (finite element/difference) calculations are needed in $2 \mathrm{D}$ and no explicit results exist.

[17] Note that Eq. (3) also exists in 2D acoustic/EM (for TE mode) systems, if $\frac{1}{h}$ is replaced by $\rho / \varepsilon$ (density/ permittivity), $\frac{1}{g}$ by $\kappa / \mu$ (compressibility/permeability), and $\eta$ by $P / H_{z}$ (pressure/magnetic field). So our cylinder systems maps to rigid/metal cylinder arrays in air/vacuum for acoustic/TE waves.

[18] N. A. Nicorovici et al., Phys. Rev. Lett. 75, 1507 (1995); P. Halevi et al., ibid. 86, 3211 (2001); N. A. Nicorovici et al., ibid. 86, 3212 (2001).

[19] F. Cervera et al., Phys. Rev. Lett. 88, 023902 (2002); A. A. Krokhin et al., ibid. 91, 264302 (2003).

[20] C. C. Mei, The Applied Dynamics of OceanSurface Waves (World Scientific, Singapore, 1989).

[21] The CPA method was originally developed for solid state physics for treating the electronic structure of random alloy systems. CPA has also been generalized to deal with EM waves (usually for random systems).

[22] Homogenization and Effective Moduli of Materials and Media, edited by J. L. Ericksen et al. (Springer-Verlag, New York, 1986); P. Sheng, Introduction to Wave Scattering, Localization, and Mesoscopic Phenomena (Academic Press, New York, 1995).

[23] It is the main approximation in our homogenization procedure that a circular cell (rather than a square unit cell) is considered for the convenience of analytic derivation.

[24] M.E. McCormick, Ocean Wave Energy Conversion (John Wiley \& Sons, New York, 1981); Wave Energy Conversion, edited by J. Brooks (Elsevier, Boston, 2003).

[25] A water-wave lens based on the conventional refraction by varying depth has been proposed by E. Mehlum [24]. 